\title{
RESEARCH
}

Open Access

\section{Inhibiting HMGB1-RAGE axis prevents pro- inflammatory macrophages/microglia polarization and affords neuroprotection after spinal cord injury}

Hong Fan ${ }^{1,2+}$, Hai-Bin Tang ${ }^{3+}$, Zhe Chen ${ }^{4+}$, Hu-Qing Wang ${ }^{1}$, Lei Zhang ${ }^{1}$, Yu Jiang ${ }^{1}$, Tao Li ${ }^{1}$, Cai-Feng Yang ${ }^{4}$, Xiao-Ya Wang ${ }^{1}$, Xia Li ${ }^{5}$, Sheng-Xi Wu ${ }^{2^{*}}$ and Gui-Lian Zhang ${ }^{1 *}$

\begin{abstract}
Background: Spinal cord injury (SCl) favors a persistent pro-inflammatory macrophages/microglia-mediated response with only a transient appearance of anti-inflammatory phenotype of immune cells. However, the mechanisms controlling this special sterile inflammation after $\mathrm{SCl}$ are still not fully elucidated. It is known that damage-associated molecular patterns (DAMPs) released from necrotic cells after injury can trigger severe inflammation. High mobility group box 1(HMGB1), a ubiquitously expressed DNA binding protein, is an identified DAMP, and our previous study demonstrated that reactive astrocytes could undergo necroptosis and release HMGB1 after SCl in mice. The present study aimed to explore the effects and the possible mechanism of HMGB1on macrophages/microglia polarization, as well as the neuroprotective effects by HMGB1 inhibition after SCI.

Methods: In this study, the expression and the concentration of HMGB1 was determined by qRT-PCR, ELISA, and immunohistochemistry. Glycyrrhizin was applied to inhibit HMGB1, while FPS-ZM1 to suppress receptor for advanced glycation end products (RAGE). The polarization of macrophages/microglia in vitro and in vivo was detected by qRT-PCR, immunostaining, and western blot. The lesion area was detected by GFAP staining, while neuronal survival was examined by Nissl staining. Luxol fast blue (LFB) staining, DAB staining, and western blot were adopted to evaluate the myelin loss. Basso-Beattie-Bresnahan (BBB) scoring and rump-height Index (RHI) assay was applied to evaluate locomotor functional recovery.

(Continued on next page)
\end{abstract}

\footnotetext{
*Correspondence: shengxi@fmmu.edu.cn; zhgl_2006@126.com

${ }^{+}$Hong Fan, Hai-Bin Tang and Zhe Chen contributed equally to this work.

${ }^{2}$ Institute of Neurosciences, Fourth Military Medical University, Xi'an 710032,

Shaanxi, China

'Department of Neurology, The Second Affiliated Hospital of Xi'an Jiaotong University, Xi'an 710004, Shaanxi, China

Full list of author information is available at the end of the article
}

(c) The Author(s). 2020 Open Access This article is licensed under a Creative Commons Attribution 4.0 International License, which permits use, sharing, adaptation, distribution and reproduction in any medium or format, as long as you give appropriate credit to the original author(s) and the source, provide a link to the Creative Commons licence, and indicate if changes were made. The images or other third party material in this article are included in the article's Creative Commons licence, unless indicated otherwise in a credit line to the material. If material is not included in the article's Creative Commons licence and your intended use is not permitted by statutory regulation or exceeds the permitted use, you will need to obtain permission directly from the copyright holder. To view a copy of this licence, visit http://creativecommons.org/licenses/by/4.0/ The Creative Commons Public Domain Dedication waiver (http://creativecommons.org/publicdomain/zero/1.0/) applies to the data made available in this article, unless otherwise stated in a credit line to the data. 
(Continued from previous page)

Results: Our data showed that HMGB1 can be elevated and released from necroptotic astrocytes and HMGB1 could induce pro-inflammatory microglia through the RAGE-nuclear factor-kappa B (NF-kB) pathway. We further demonstrated that inhibiting HMGB1 or RAGE effectively decreased the numbers of detrimental pro-inflammatory macrophages/ microglia while increased anti-inflammatory cells after SCI. Furthermore, our data showed that inhibiting HMGB1 or RAGE significantly decreased neuronal loss and demyelination, and improved functional recovery after SCl.

Conclusions: The data implicated that HMGB1-RAGE axis contributed to the dominant pro-inflammatory macrophages/ microglia-mediated pro-inflammatory response, and inhibiting this pathway afforded neuroprotection for $\mathrm{SCl}$. Thus, therapies designed to modulate immune microenvironment based on this cascade might be a prospective treatment for SCl.

Keywords: Spinal cord injury, Macrophages/microglia, HMGB1, Polarization, RAGE

\section{Introduction}

Spinal cord injury (SCI) initiates primary injury and a cascade of secondary injury, leading to transient or permanent functional disorder even paralysis [1]. Preservation of motor function after SCI is one of the most concerned issues during treatment and rehabilitation, which is mainly depended on the neuronal survival and myelin maintenance $[2,3]$. That the relationship between neuronal or myelin loss and pro-inflammatory response after SCI has been proven by other research and our previous study $[4,5]$. SCI triggers a dominant pro-inflammatory response, and resident microglia and recruited macrophages are central players in innate immune response following SCI $[6,7]$. However, the pathological mechanism of the pro-inflammatory macrophages/microglia-mediated detrimental microenvironment after SCI has been not fully clarified. Thus, revealing and regulating the underlying mechanism might be a prospective strategy to promote tissue repair and functional recovery after SCI.

In trauma-induced sterile inflammation, molecules released from dead cells play pivotal roles in initialization of immune activation [8]. It was well known that apoptosis within normal physiological or pathological process is generally silent, and apoptotic cells can be swiftly removed without immune activation [9]. However, necrosis after physical trauma, infection, or toxic insult activates innate immunity [10]. Damage-associated molecular patterns (DAMPs) released from necrotic cells after injury have been proved as a key to initiate and accelerate production of pro-inflammatory mediators [11].

High mobility group box 1(HMGB1), a highly preserved nuclear DNA-binding protein, is one of the defined DAMPs [12]. As HMGB1 is expressed in all nucleated animal cells, it can be served as a powerful mediator of inflammation when passively released from necrotic cells [13]. HMGB1 has been found to participate in many immune-related diseases of the central nervous system (CNS), including traumatic brain injury, stroke, epilepsy, multiple sclerosis, and SCI [14-18]. Our previous study had demonstrated that reactive astrocytes could undergo receptor-interacting serine-threonine kinase 3 (RIP3)/mixed lineage kinase domain-like protein (MLKL)-mediated necroptosis, a programmed necrosis, and release HMGB1after SCI in mice [19]. However, it is not fully understood on how HMGB1 participated in polarization of pro-inflammatory macrophages/microglia and its related pro-inflammatory response after SCI.

The present study was designed to explore the effects and the underlying mechanism of HMGB1 on macrophages/microglia polarization, as well as its effects on neuronal and myelin loss after SCI. Both of the elevated HMGB1 concentration in serum and increased cytoplasmic HMGB1 in injured cord were found in our SCI model of rats. Our in vitro study further demonstrated that HMGB1 could polarize microglia to pro-inflammatory phenotype through receptor for advanced glycation end products (RAGE)-nuclear factor-kappa B (NF-kB) pathway. By application of glycyrrhizin, an inhibitor of HMGB1 or FPS-ZM1, an inhibitor of RAGE, we further found that inhibiting HMGB1 or RAGE decreased the numbers of pro-inflammatory macrophages/microglia while increased the numbers of anti-inflammatory cells. Furthermore, we found that inhibiting RAGE effectively enhanced neuronal survival and myelin preservation after SCI. Moreover, inhibiting both of HMGB1 and RAGE significantly improved functional recovery after SCI. Taken together, these data suggested that inhibiting HMGB1RAGE axis prevented pro-inflammatory macrophages/ microglia polarization and afforded neuroprotection after $\mathrm{SCI}$ in rats. It might be a prospective strategy for clinical SCI treatment based on HMGB1-RAGE axis.

\section{Materials and methods}

\section{Animal grouping and treatment}

A total of 150 male Sprague Dawley rats (about $250 \mathrm{~g}$ ) were purchased from the Animal Center of Xi'an Jiaotong University. Experimental protocols of the animals in this study were approved by Animal Care and Use Committee of Xi'an Jiaotong University. SD rats were randomized into 
four groups: sham control group, $\mathrm{SCI}+$ saline group, $\mathrm{SCI}$ + glycyrrhizin (Sigma-Aldrich, G2137) group, and SCI + FPS-ZM1 (MedChemExpress, HY-19370) group. Rats in the sham control group received laminectomy only, while $0.5 \mathrm{~mL}$ of sterile saline was administrated via intraperitoneal injection (i.p.) after SCI for the SCI + saline group. According to previous researches and our pilot data [2022], $10 \mathrm{mg} / \mathrm{kg}$ of glycyrrhizin or $1 \mathrm{mg} / \mathrm{kg}$ of FPS-ZM1 in saline was daily administered via i.p. for 14 days after SCI for the SCI + glycyrrhizin group and the SCI + FPS-ZM1 group, respectively, with the first injection immediately after SCI.

\section{$\mathrm{SCl}$ model}

The SCI was established according to the modified Tetzlaff spinal cord lateral crush model as we described previously $[23,24]$. Rats were anesthetized with $10 \%$ chloral hydrate $(400 \mathrm{mg} / \mathrm{kg})$, followed by laminectomy of vertebrae T8 to expose the spinal cord. The spinal cord crush model was made by the forceps (53327T, 66 Vision-Tech Co., Ltd., China) mounted on our designed mechanical device. The cord was clamped by forceps with $0.5 \mathrm{~mm}$-width when fully closed, and the crush remained for $20 \mathrm{~s}$. Rats in sham group underwent laminectomy only. After operation, urination was aided once a day until reestablishment of micturition reflex.

\section{Tissue processing}

At designated time points after injury, rats were anesthetized and perfused intracardially with $200 \mathrm{ml}$ of $0.01 \mathrm{M}$ PBS (pH 7.4), followed by $400 \mathrm{ml}$ of $4 \%$ paraformaldehyde (PFA). Two-centimeter segments of fixed tissues containing the lesion site were obtained and cryoprotected in 30\% sucrose for $48 \mathrm{~h}$. After embedded in OCT, both of serial transverse sections (10 $\mu \mathrm{m}$ thick) and serial sagittal sections $(12 \mu \mathrm{m}$ thick) were cut within a Leica CM1950 cryostat. The slides were collected and stored at $-20{ }^{\circ} \mathrm{C}$ for staining.

\section{Immunohistochemistry Immunoflurence}

After air-dried for $2 \mathrm{~h}$, slides were blocked in $0.01 \mathrm{M}$ PBS containing 3\% BSA and $0.1 \%$ Triton X-100 for $1 \mathrm{~h}$ and then incubated with primary antibodies overnight at $4{ }^{\circ} \mathrm{C}$. The primary antibodies used in tissue staining were rabbit anti-HMGB1 (1:500, Proteintech), mouse antiglial fibrillary acidic protein (GFAP, 1:2000, SigmaAldrich), rabbit anti-TLR4 (1:500, Abcam), goat antiIba-1 (1:500, Abcam), rabbit anti-iNOS (1:200, Abcam), and rabbit anti-Arginase-1 (1:100, Santa Cruz). Antigen retrieval was performed with citrate buffer before HMGB1-staining. After washing three times with PBS, the slides were incubated with Alexa Fluor 594-labeled or Alexa Fluor 488-labeled corresponding secondary antibodies (1:500, Jackson ImmunoResearch Laboratories) in the dark for $2 \mathrm{~h}$. Nuclei were counterstained with Hoechst 33342.

\section{DAB-staining}

After fixation with acetone, the slides were incubated in $0.3 \% \mathrm{H}_{2} \mathrm{O}_{2}$ solution in PBS at room temperature for 10 min to block endogenous peroxidase activity. The slides were then in turn incubated with rabbit anti-MBP (1: 200, Abcam), biotinylated secondary antibody, and Avidin-Biotin Complex (ABC)-HRP conjugates. DAB substrate $\left(0.05 \% \mathrm{DAB}-0.015 \% \mathrm{H}_{2} \mathrm{O}_{2}\right.$ in $\left.\mathrm{PBS}\right)$ solution was subsequently applied to reveal the color of the antibody staining. The slides were dehydrated through 4 changes of alcohol $(95,95,100$, and 100\%), followed with 3 changes of xylene.

\section{Immunocytoflurence}

For immunocytochemistry, cells were fixed in 4\% PFA for $30 \mathrm{~min}$. After blocking in 3\% BSA without Triton X100 for $30 \mathrm{~min}$, the cells were stained with the following primary antibodies: rabbit anti-RAGE antibody (1:200, Proteintech), rat anti-F4/80 (1:200, Bio-Rad), and rabbit anti-iNOS (1:200, Abcam). Cells were then incubated with the corresponding secondary antibodies. Sections were examined and photographed under a confocal microscope (LSM 800, Zeiss).

\section{Enzyme-linked immunosorbent assay (ELISA)}

The concentrations of HMGB1 in serum were measured by ELISA. Rat serum was sampled, and HMGB1 levels were then determined using an ELISA kit (IBL) according to the manufacturer's instructions. Absorbance at $450 \mathrm{~nm}$ was measured by multimode microplate reader (TECAN, infinite M200).

\section{Quantitative real-time polymerase chain reaction (qRT- PCR)}

Total RNA was isolated from cultured cells or from 1.5 $\mathrm{cm}$ length of injured cord segments (or from uninjured cord) using TRizol (Promega). After reverse transcription using a reverse transcriptase kit (TaKaRa), we performed real-time PCR for HMGB1, iNOS, IL-4, IL-10, IL-12, IL-18, CD86, CD206, TNF $\alpha$, Arginase1, and Ym1 with SYBR green (TaKaRa) on Bio-Rad PCR system (Bio-Rad). The sequences of primers utilized are listed in Table 1. Gene expression levels are calculated by the $2^{-\triangle \Delta C T}$ method and the data were presented normalized by GAPDH.

\section{Microglial cell culture and treatment}

Microglial cells were obtained from neonatal (about 3day-old) SD rats, as described previously [4]. Microglial cells were cultured in DMEM supplemented with 3\% 
Table 1 PCR primer sequences

\begin{tabular}{|c|c|c|}
\hline Gene & Forward primer( $\left(5^{\prime}-3^{\prime}\right)$ & Reverse primer $\left(5^{\prime}-3^{\prime}\right)$ \\
\hline HMGB1 & GCCCATTTGGGTCACATGG & TGCAGGGTGTGTGGACAAAA \\
\hline iNOS & AGAGACGCTTCTGAGGTTCC & CTGCACCAACTCTGCTGTTC \\
\hline $\mid \mathrm{L}-4$ & TCCACGGATGTAACGACAGC & TGGTGTTCCTTGTTGCCGTA \\
\hline $\mid \mathrm{L}-10$ & CCTCTGGATACAGCTGCGAC & GTAGATGCCGGGTGGTTCAA \\
\hline IL-12 & ATCATCAAACCGGACCCACC & CAGGAGTCAGGGTACTCCCA \\
\hline IL-18 & ACCGCAGTAATACGGAGCAT & TCTGGGATTCGTTGGCTGTT \\
\hline CD86 & CGTCAAGACATGTGTAACCTGC & ACCGACTIITTCCGGTCCTG \\
\hline CD206 & TCAACTCTTGGACTCACGGC & CATGATCTGCGACTCCGACA \\
\hline TNFa & ACTGAACTTCGGGGTGATCG & TGGTGGTTTGCTACGACGTG \\
\hline Arginase1 & ACAAGACAGGGCTACTITCAGG & ACAAGACAAGGTCAACGCCA \\
\hline Ym1 & AGTTTGGATCTGCCCCGTTC & TTAGGAGGGCTTCCACGAGA \\
\hline GAPDH & AGTGCCAGCCTCGTCTCATA & GGTAACCAGGCGTCCGATAC \\
\hline
\end{tabular}

FBS, penicillin-streptomycin $(100 \mathrm{U} / \mathrm{ml}-100 \mu \mathrm{g} / \mathrm{ml})$ and $4 \mathrm{mM}$ L-glutamine. Cells were grown on poly-L-lysine coated slides in 6-well plates. For HMGB1 and FPSZM1 treatment, 0.4 or $1 \mu \mathrm{g} / \mathrm{ml}$ of HMGB1 and $1 \mu \mathrm{M}$ of FPS-ZM1 were added to the culture medium. At the end of treatment, total RNAs were isolated for qRT-PCR, total cellular proteins were isolated for Western-blotting, or cells were harvested for immunostaining.

\section{Western blotting}

Whole cell lysates were prepared from $1.5-\mathrm{cm}$-long segments of injured cord containing the injury site or microglia. Protein were isolated and homogenized with ice-cold radioimmunoprecipitation assay (RIPA) buffer. Proteins were resolved on $10 \%$ sodium dodecyl sulfatepolyacrylamide gel (SDS-PAGE) gel and transferred onto a polyvinylidene difluoride (PVDF) membrane. Blots were incubated with primary antibodies for iNOS (1:500, Abcam), CD86 (1:600, Proteintech), MBP (1:1000, Milipore), RAGE (1:500, Proteintech), phospho-NF$\kappa B$ p65/p-P65 (1:1000, Cell Signaling), or beta-actin (1:5000, Sigma) at $4{ }^{\circ} \mathrm{C}$ overnight. After incubation with corresponding horseradish peroxidase-conjugated secondary antibodies (1:5000, Jackson ImmunoResearch) at RT for $1 \mathrm{~h}$, the bands were visualized using a Bio-Rad Image Lab system, and densitometry analysis was performed with the Image J software.

\section{Luxol fast blue staining}

Luxol fast blue (LFB) staining was performed as described previously [4]. Serial transverse cryosections (12 $\mu \mathrm{m}$ thickness) were incubated in $0.1 \%$ Luxol fast blue (Sigma) in acidified 95\% ethanol overnight at $60{ }^{\circ} \mathrm{C}$. Differentiation and counterstaining was performed with $0.05 \%$ lithium carbonate and cresyl violet solution. LFBstained tissue sections were examined by light microscopy and analyzed for myelin sparing.

\section{Nissl staining}

Nissl staining was performed to assess neuronal survival. The sections were rinsed in deionized water, dipped in a warm $\left(50{ }^{\circ} \mathrm{C}\right)$ solution of $1 \%$ thionine for $45 \mathrm{~min}$, and differentiated with $70 \%$ alcohol for about $5 \mathrm{~min}$.

\section{Behavioral evaluation \\ BBB scores}

The Basso-Beattie-Bresnahan (BBB) scores [25] were used to evaluate functional recovery at 1 day before and $1,3,7,10,14$, and 21 days after SCI. The tests were performed by two independent and blinded observers.

\section{Rump-height index (RHI) assay}

Rats were video-recorded from the left to the right side during walking on a runway bar $(150 \mathrm{~cm}$ long, $8 \mathrm{~cm}$ wide, and $2 \mathrm{~cm}$ thick) before injury and at $1,3,7,10,14$, and $21 \mathrm{dpi}$ [26]. The RHI is defined as the height of the rump, normalized to the thickness of the beam, measured along the same vertical line. The standardized RHI (dividing post-injury value by pre-injury value) was introduced to minimize the variations of pre-surgery RHI of each animal as we used previously [27].

\section{Image analysis}

The sections of GFAP staining, Nissl staining, and LFB staining were captured and examined using a fluorescence microscope (BX51, Olympus). The lesion area delineated by GFAP immunohistochemistry was calculated by Photoshop CS3 and converted to the physical size based on microscope calibration. The other immunostained sections were photographed under a confocal microscope (LSM 800, Zeiss).

For cell counting, all of the positive cells in the defined area in randomly chosen slide were counted. Then, calibration of the profile numbers was adopted to minimize the unbiased data as recommended [28, 29]. 


\section{Statistics}

Quantification was performed by researchers who were blinded to the experiment design. Data are expressed as mean \pm standard error of the mean (SEM). The Student's t-test was used for comparisons between two groups. For multiple comparisons, the data were analyzed by one-way ANOVA followed by Tukey post-hoc tests. Assessment of BBB scales and RHI was analyzed by two-way RM ANOVA. P $<0.05$ was considered statistically significant.

\section{Results}

\section{Increased expression and release of HMGB1 after SCI}

We first examined the expression changes of HMGB1 after SCI, one potential mediator from the necrotic core of injured cord, and the data of qPCR showed that the mRNA level of HMGB1 increased significantly from 1 to $21 \mathrm{dpi}$, with the highest level at $3 \mathrm{dpi}\left({ }^{*} P<0.05\right.$, ${ }^{* *} P$ $<0.01$, Fig. 1a). Immunohistochemistry detected cytoplasmic, nuclear, and both cytoplasmic and nuclear HMGB1-immunoreactivity around lesion center (Fig. 1b), and quantification showed that approximately $60 \%$ of the HMGB1-positive cells were GFAP-positive (Fig. 1c), which was consistent with our previous result in mice of SCI [19]. Further data showed that percentage of cytoplasmic HMGB1 in GFAP-positive cells gradually increased and reached peak $(67.0 \pm 7.56 \%)$ at $7 \mathrm{dpi}$, percentage of both cytoplasmic and nuclear HMGB1-immunoreactivity gradually decreased from $1 \mathrm{dpi}$, and lowest percentage (10.2 \pm $1.81 \%$ ) of nuclear HMGB1 was found at $7 \mathrm{dpi}$ (Fig. 1d). As it was reported that HMGB1 functions as a DAMP to induce a pro-inflammatory response when it is passively released from the necrotic cells [30], we tested the serum HMGB1 levels by ELISA after SCI. The result showed that the concentration of HMGB1 was significantly increased in serum at 3 days after SCI, when compared to shamoperated controls (Fig. 1e). These data suggested that the released HMGB1 might be a candidate DAMP to contribute to the persistent pro-inflammatory response after SCI.

\section{HMGB1 induced pro-inflammatory polarization of microglial cells}

Although HMGB1 was reported to induce proinflammatory response after SCI [31], there still lacks direct evidence of HMGB1 participating in polarization of pro-inflammatory microglia. To determine the effect of HMGB1 on polarization of microglia, we examined the mRNA levels of pro-inflammatory or anti-inflammatory phenotypic markers in primary-cultured microglia upon HMGB1 treatment. The data showed that mRNA levels of TNF $\alpha$, iNOS, and CD86 were significantly increased in microglia treated with 0.4 or $1 \mu \mathrm{g} / \mathrm{ml}$ HMGB1, compared to cells under normal condition $\left({ }^{* * *} P<0.01\right.$, $* * * * 0.001$, Fig. $2 \mathrm{a}-\mathrm{c}$ ), while levels of IL-12 and IL-18, another two pro-inflammatory markers, showed no significant changes upon HMGB1 treatment (Fig. 2d-e). Nevertheless, no significant changes in anti-inflammatory markers Arg1, CD206, and IL-4 were observed after HMGB1 treatment (Fig. 2f-h), except for a decrease of IL-10 ( $\mathrm{*}<0.05$, Fig. 2i). The above data provided direct evidence for HMGB1 in polarizing microglia to pro-inflammatory state.

\section{RAGE mediated the HMGB1-induced pro-inflammatory polarization of microglia}

Considering that HMGB1 could function as a DAMP through receptors TLR4 or RAGE during distinct conditions [32], we then investigated the specific receptor involved in pro-inflammatory polarization of macrophages/ microglia upon HMGB1 treatment. The data of qPCR showed that HMGB1 increased both mRNA levels of TLR4 and RAGE in microglia (Fig. 3a-b). Although that immunostaining showed that TLR4 was mainly expressed by GFAP-positive cells $5 \mathrm{dpi}$, with only $17 \%$ of TLR4positive were Iba-1-positive (Fig. $3 c-d$, Additional file 1: Figure S1), the possibility that activation of TLR4 on astrocytes might influence macrophage phenotype through a paracrine method could not be excluded. Further, immunohistochemistry showed that the positive staining of RAGE was mainly co-localized with F4/80-positive macrophages/microglia after SCI. Furthermore, we found that glycyrrhizin, an inhibitor of HMGB1 could decrease the percentage of RAGE-positive cells in F4/80-positive cells after SCI (Fig. 3e-f). The above results suggested that RAGE was a possible receptor for pro-inflammatory polarization of microglia upon HMGB1 treatment.

Our immunocytochemistry data revealed that the numbers of RAGE-positive cells and its immunofluorescence intensity (IFI) were significantly increased in microglia upon HMGB1 treatment in vitro $(* * \% P<0.001$, Fig. $4 \mathrm{a}-\mathrm{c})$. The data of western blot also showed that HMGB1 induced expression of RAGE in microglia ( ${ }^{*} \mathrm{P}<0.01$, Fig. $\left.4 \mathrm{~d}-\mathrm{e}\right)$. As 1 $\mu \mathrm{g} / \mathrm{ml}$ of HMGB1 exerted a stronger inducible effect on expression of pro-inflammatory markers and RAGE, this concentration of HMGB1 was adopted for further experiments.

We further applied FPS-ZM1, a RAGE-specific blocker to examine whether RAGE was involved in HMGB1induced pro-inflammatory polarization of the microglia. Our data showed that FPS-ZM1 significantly reversed the increased mRNA of iNOS, CD86, and TNF $\alpha$ induced by HMGB1 $(* P<0.05, * * P<0.01, * * * P<0.001$, Fig. $5 a-$ c). The result of immunostaining also showed that FPSZM1 significantly decreased the numbers of iNOSpositive cells induced by HMGB1 ( ${ }^{*} P<0.05$, * ${ }^{*} P<0.01$, Fig. $5 \mathrm{~d}-\mathrm{e})$. The data of western blot further showed that FPS-ZM1 could reduce the expression of RAGE and its downstream molecule $\mathrm{p}-\mathrm{NF}-\mathrm{\kappa B}$ induced by HMGB1 ( $P$ $<0.05, * * P<0.01$, Fig. $5 f-g)$. The above data indicated 
A

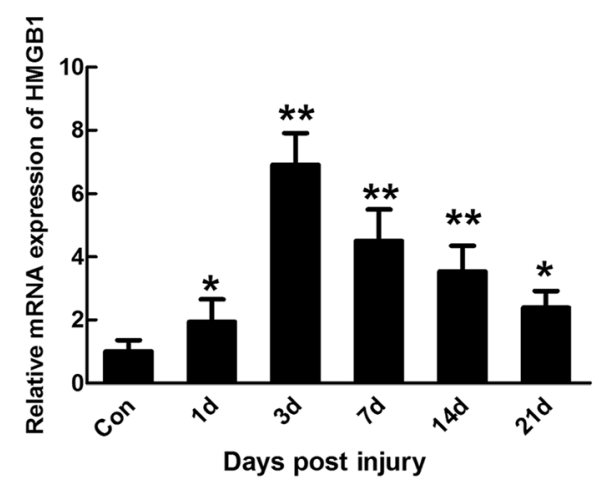

E

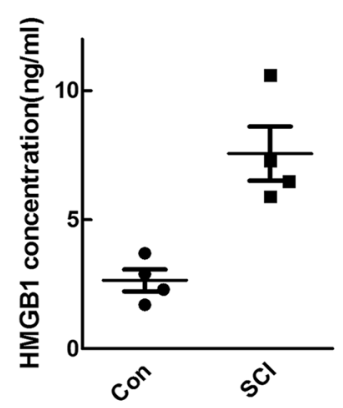

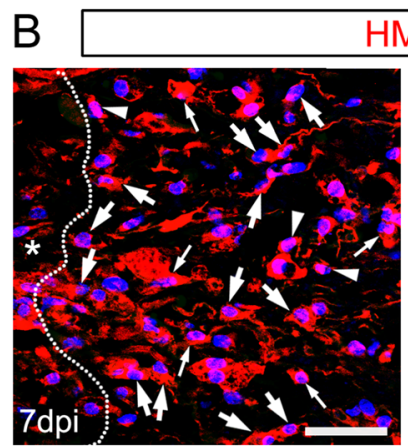

C

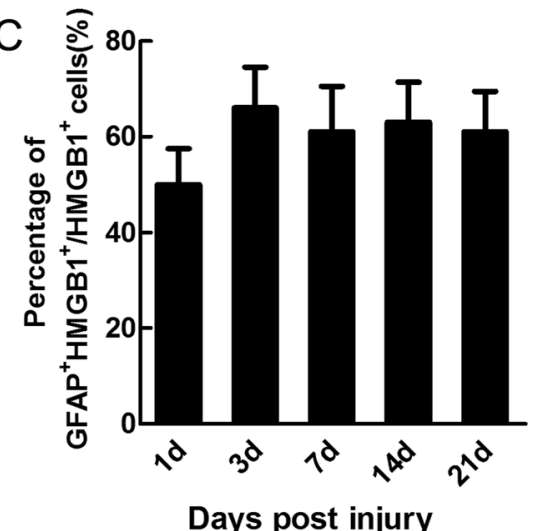

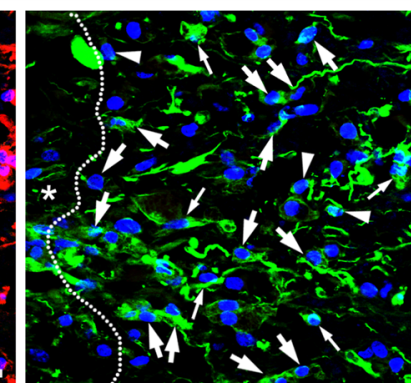

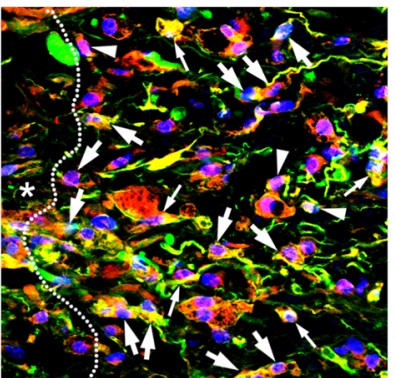

D ᄃ nuclear

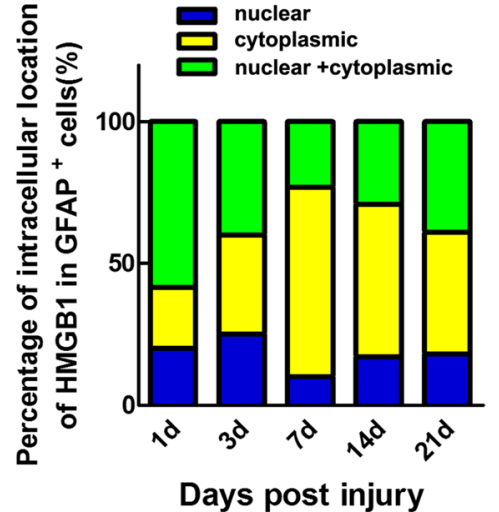

Fig. 1 HMGB1 Expression after SCI. a Temporal pattern of HMGB1 mRNA in spinal cord of sham-operated and SCI rats. Note that the mRNA level of HMGB1 was significantly increased from 3 to $21 \mathrm{dpi}$. Results were expressed as mean \pm SEM of 3 rats, ${ }^{*} P<0.05$, ** $P<0.01$. b Immunohistochemistry of HMGB1. Note that all the cells around the epicenter were labeled with HMGB1-positive signal, with different intracellular localization (nuclear indicated by arrowheads, cytoplasmic indicated by thick arrows, and both cytoplasmic and nuclear indicated by thin arrows). Asterisks indicate the epicenter. Scale bar $=80 \mu \mathrm{m}$. c-d Quantification of intracellular localization of HMGB1. About 60\% of the HMGB1-positive cells were GFAP-positive. Note that percentage of cytoplasmic HMGB1 in GFAP-positive cells reached peak (67.0 $\pm 7.56 \%)$ at 7 dpi, and lowest percentage (10.2 $\pm 1.81 \%)$ of nuclear HMGB1 at 7 dpi. e Elevated HMGB1 serum levels in 4 rats 3 days after $\mathrm{SCl}$ compared with sham-operated controls, ${ }^{*} P<0.05$

that HMGB1-induced pro-inflammatory polarization of microglia was at least partially through RAGE-NF-kB pathway.

Inhibiting HMGB1-RAGE axis influenced the polarization of macrophages/microglia after $\mathrm{SCl}$

Based on the above in vitro data, we applied glycyrrhizin and FPS-ZM1 to determine the effects of HMGB1-RAGE inhibition on polarization of macrophage/microglia after SCI. The data showed that both glycyrrhizin and FPS-
ZM1 significantly decreased the mRNA levels of proinflammatory markers of iNOS, IL-12, CD86, and TNF $\alpha$ $\left({ }^{*} P<0.05\right.$, Fig. $\left.6 \mathrm{a}-\mathrm{d}\right)$ at 14 days after injury. Further, immunostaining showed that the number of iNOSexpressing macrophages/microglia in bilateral areas 200 $\mu \mathrm{m}$ rostral and dorsal to lesion center was significantly decreased in FPS-ZM1-treated rats $\left({ }^{*} P<0.05\right.$, Fig. 6e-f), while no significant differences in total number of macrophage/ microglia between saline and FPS-ZM1 group (data not shown). Furthermore, the data of western blot showed 

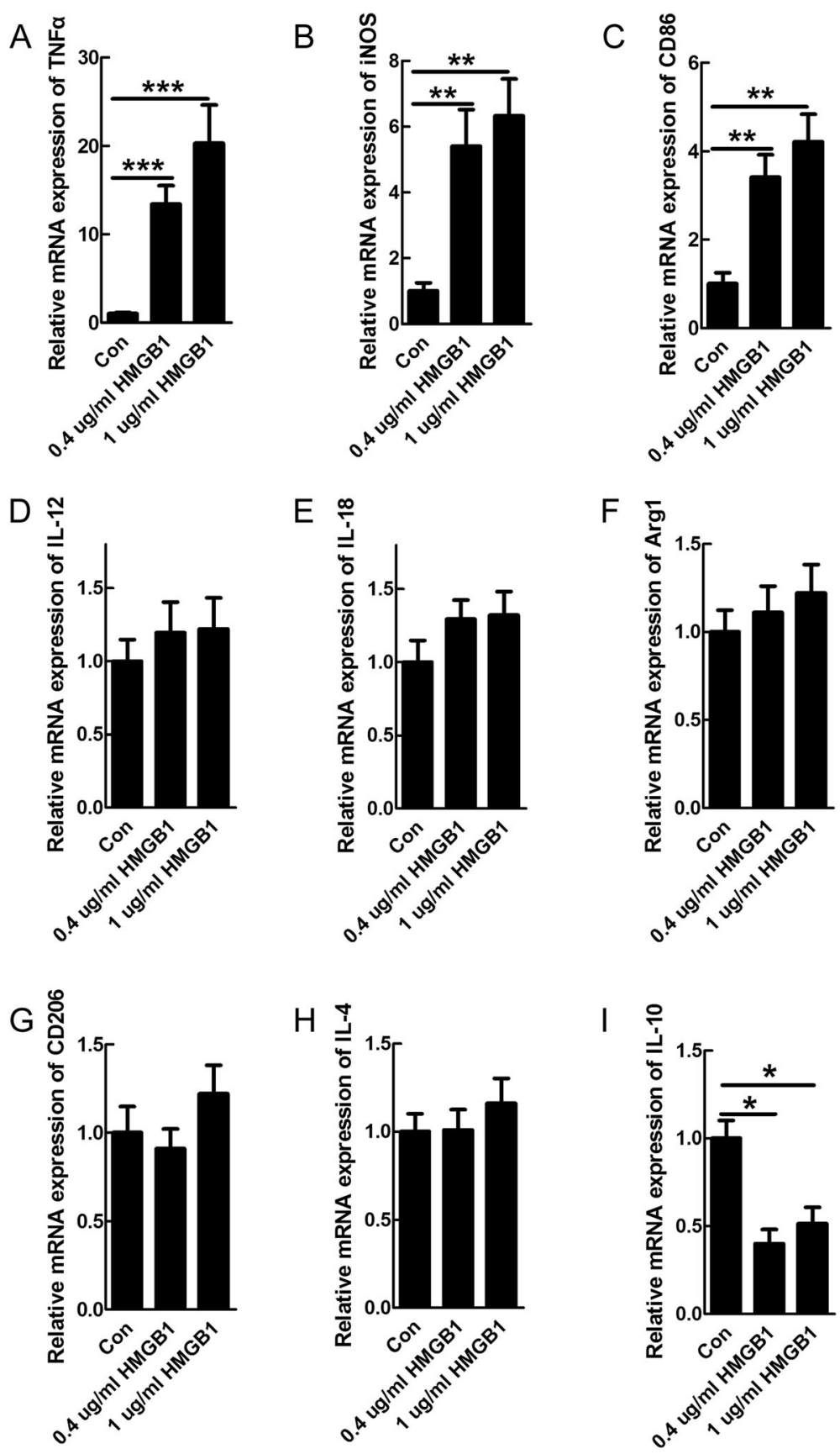

Fig. 2 HMGB1 increased the expression of pro-inflammatory markers in the microglia. The mRNA expression of pro-inflammatory markers of TNFa (a), iNOS (b), CD86 (c), and IL-12 (d) was significantly increased in microglia $24 \mathrm{~h}$ after recombinant HMGB1 treatment, except for no change of IL18 (e). No significant changes in anti-inflammatory markers of Arg1, CD206, and IL-4 after HMGB1 treatment (f-h), except for a decrease of IL-10 (i). Results are presented as mean \pm SEM. $N=3,{ }^{*} P<0.05,{ }^{* *} P<0.01,{ }^{* * *} P<0.001$

that FPS-ZM1 inhibited the injury-induced proinflammatory polarization, shown by lowered levels of iNOS and CD86 $(* P<0.05$, $* P<0.01$, Fig. $6 g-i)$.

Meanwhile, we tested the effect of HMGB1-RAGE inhibition on anti-inflammatory polarization. The data showed that both glycyrrhizin and FPS-ZM1 significantly increased the mRNA levels of Arginase1, Ym1, IL-4, and IL-10
(Additional file 2: Figure S2A-D). The immunohistochemical data further revealed that the Arginase1-positive macrophages/microglia in the epicenter was significantly increased in the FPS-ZM1 group (Additional file 2: Figure $\mathrm{S} 2 \mathrm{E}-\mathrm{F})$. In the sham-operated rats, there were none of the iNOS- or Arginase1-positive cells (data not shown). The above results demonstrated that HMGB1 or RAGE 

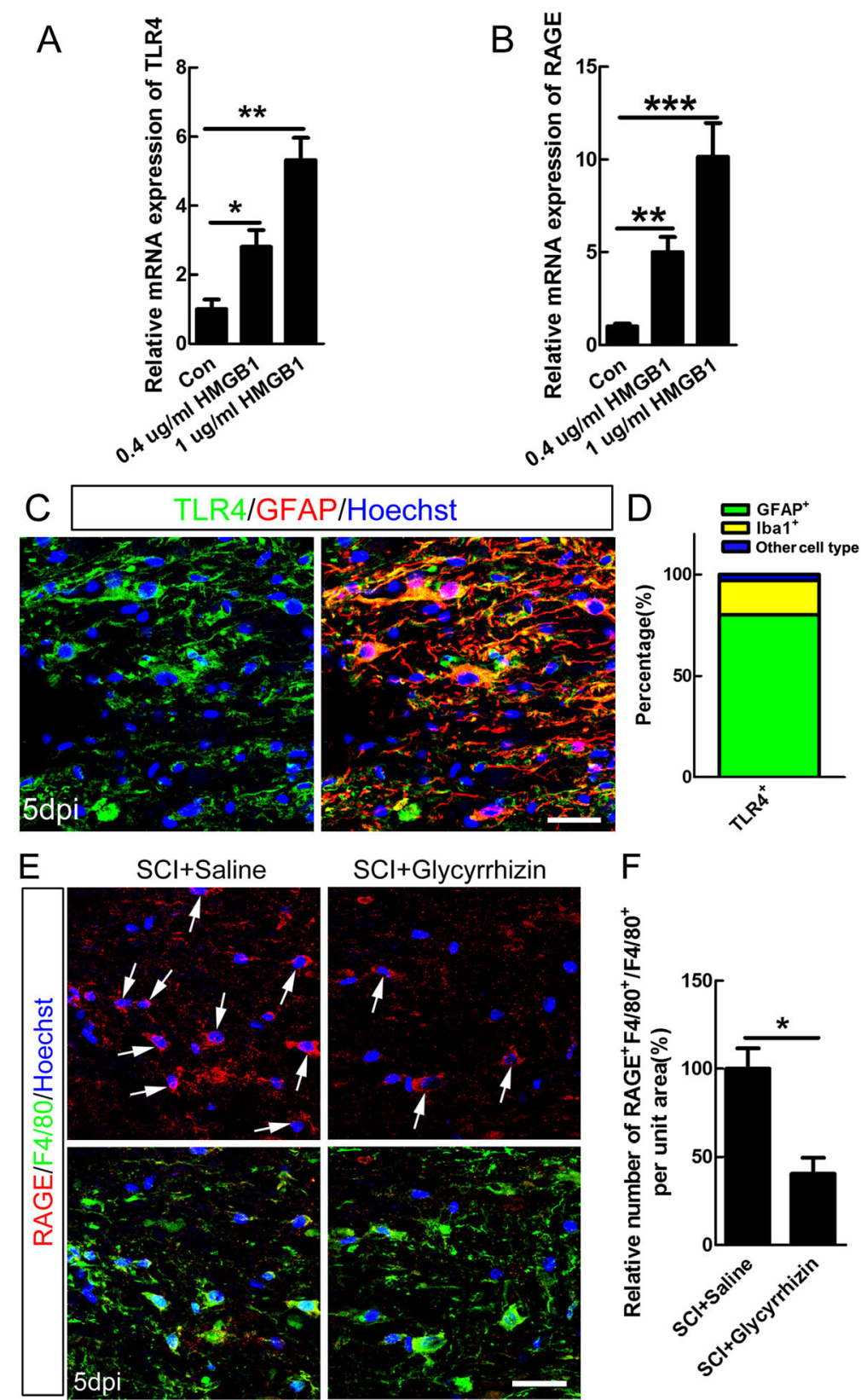

Fig. 3 HMGB1 activated TLR4 and RAGE in vitro, and SCI induced the expression of RAGE in macropgahes/microglia. The mRNA expression of TLR4 (a) and RAGE (b) was significantly increased in microglia at $24 \mathrm{~h}$ after 0.4 or $1 \mu \mathrm{g} / \mathrm{ml}$ HMGB1 treatment. (c-d) Double-staining of GFAP/TLR4 and quantification of TLR4-positive cell types at 5 dpi. Note that about 80\% TLR4-positive cells were GFAP-positive, while $17 \%$ were Iba-1 positive. Scale bar $=50 \mu \mathrm{m}$. e Representative images of double-staining of RAGE and F4/80 in injured cord of saline- or glycyrrhizin-treated rats. Scale bar $=50 \mu \mathrm{m}$. $\mathbf{f}$ Quantification of the relative percentage of RAGE-positive cells in F4/80-positive cells. $N=4,{ }^{*} P<0.05$

inhibition could suppress macrophages/microglia polarized to pro-inflammatory phenotype, while promote antiinflammatory polarization after SCI.

Inhibiting RAGE decreased lesion size and neuronal death after SCl

The special pro-inflammatory macrophages/microgliamediated detrimental response after SCI was closely related to the degree of secondary injury [33]. Considering that inhibiting HMGB1could ameliorate the proimmune environment [34], we subsequently assessed the effects of HMGB1-RAGE inhibition on lesion cavity and neuronal survival. Immunohistochemistry for GFAP was performed to evaluate the lesion size, and the result showed that FPS-ZM1 significantly reduced the lesion area at $21 \mathrm{dpi}\left({ }^{*} P<0.05\right.$, Fig. $\left.7 \mathrm{a}-\mathrm{b}\right)$. Furthermore, the 

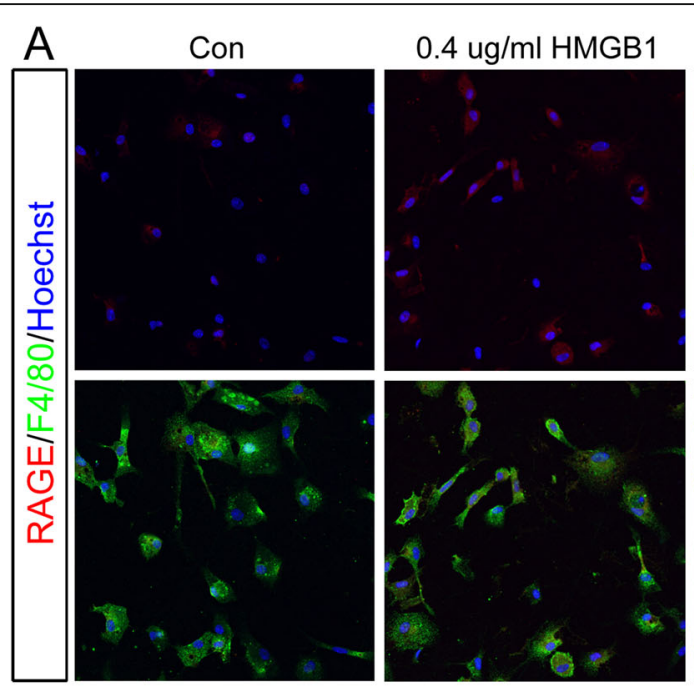

\section{$1 \mathrm{ug} / \mathrm{ml} \mathrm{HMGB} 1$}

B
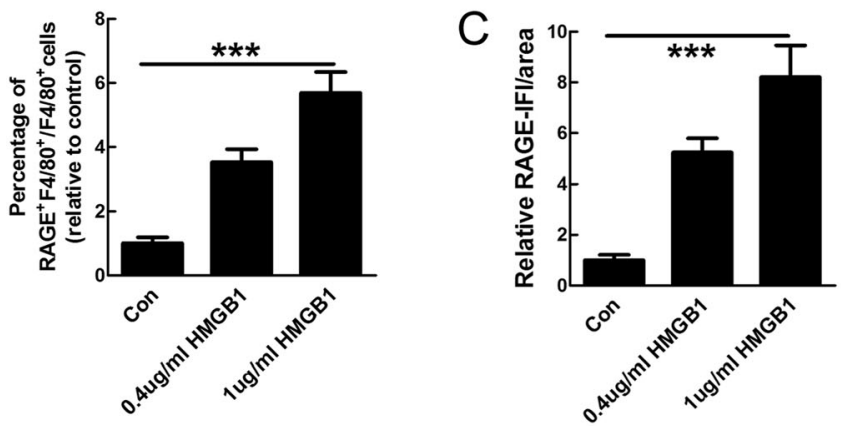

D
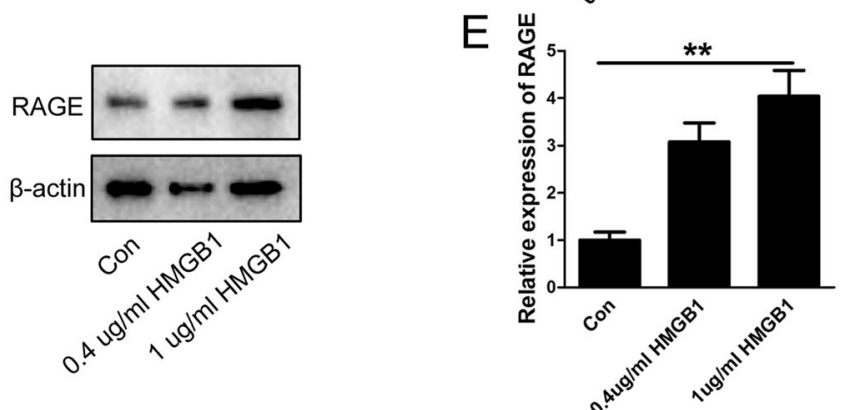

Fig. 4 HMGB1 induced RAGE expression in microglia. a Representative images of double-staining of RAGE and F4/80 in microglia under normal condition or HMGB1 treatment. Scale bar $=50 \mu \mathrm{m}$. b-c Quantification of the numbers of RAGE-positive cells and the IFI/area of RAGE. Note that both the percentage of RAGE-positive cells and IFI of RAGE were significantly increased after HMGB1 treatment. $N=3,{ }^{* *} P<0.001$. IFI, immunofluorescence intensity. $\mathbf{d}$-e Quantification of the protein expression levels of RAGE in control and HMGB1-treated group. $\beta$-actin was used as a loading control. Data were expressed as mean \pm SEM. $N=3,{ }^{* *} P<0.01$

data of Nissl staining showed that the number of Nisslstained neurons in the area of $600 \mu \mathrm{m}$ away from each side of the lesion was increased by 1.8 times in FPSZM1-treated rats at $21 \mathrm{dpi}\left({ }^{*} \mathrm{P} P<0.01\right.$, Fig. $\left.7 \mathrm{c}-\mathrm{d}\right)$.

\section{Inhibiting RAGE reduced myelin loss after SCI}

Besides neuronal survival, we also observed myelin preservation, which was another pivotal factor for locomotion recovery after SCI. LFB staining, immunostaining, and western blot were performed to assess the myelin loss. Data of LFB staining showed that FPS-ZM1 significantly decreased myelin loss at $2000 \mu \mathrm{m}$ rostral and caudal to the injury site, as well as at the epicenter ${ }^{*} \mathrm{P}<0.05$, Fig. $\left.8 \mathrm{a}-\mathrm{b}\right)$. Moreover, the intensity of MBPpositive myelin was significantly increased in FPS-ZM1treated rats $\left({ }^{* *} P<0.01, * P<0.05\right.$, Fig. $\left.8 \mathrm{c}-\mathrm{d}\right)$. The data of western blot further confirmed that the spared myelin was significantly increased after FPS-ZM1 treatment $(* \%$ $<0.01,{ }^{*} P<0.05$, Fig. 8e-f). In addition, our data from 5-bromo-2'-deoxyuridine (BrdU) incorporation assay showed that FPS-ZM1 had no significant effect on oligodendrocyte regeneration after SCI (data not shown). The 
A
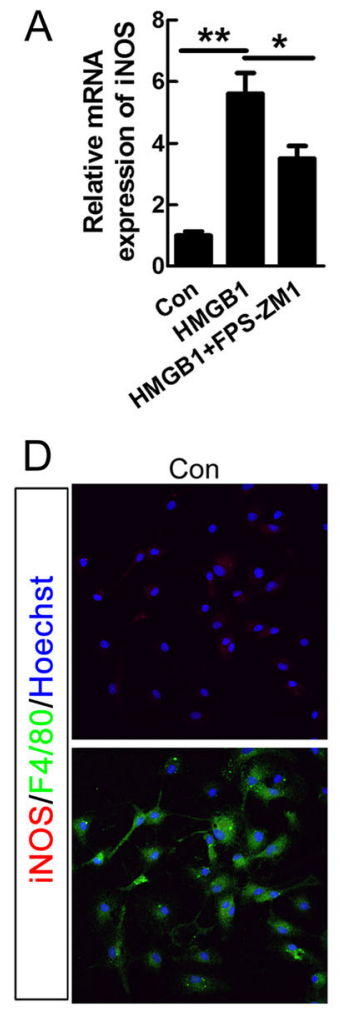

$\mathrm{F}$

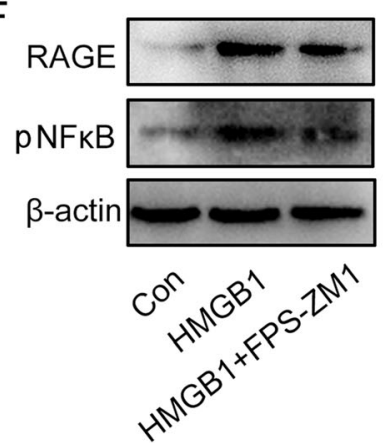

$\mathrm{B}$
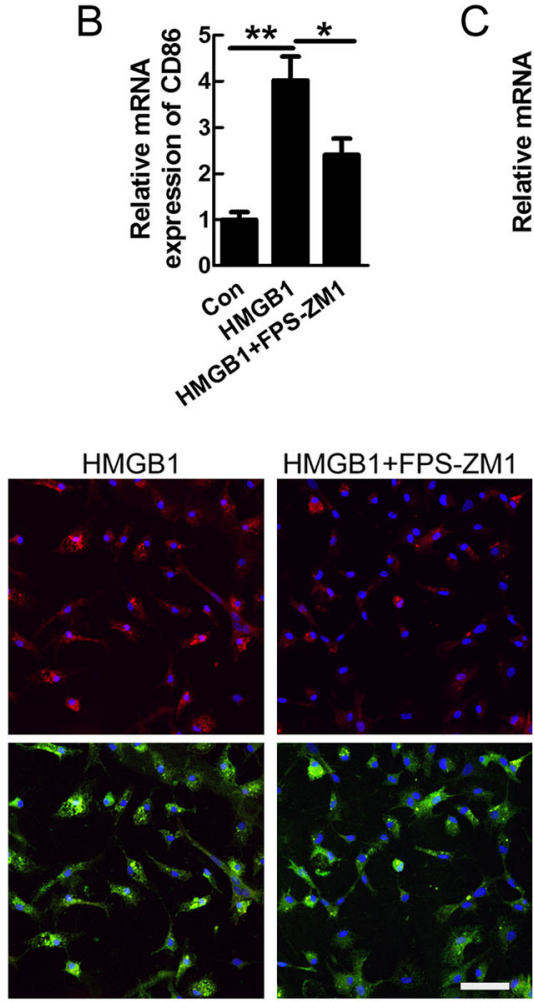

G
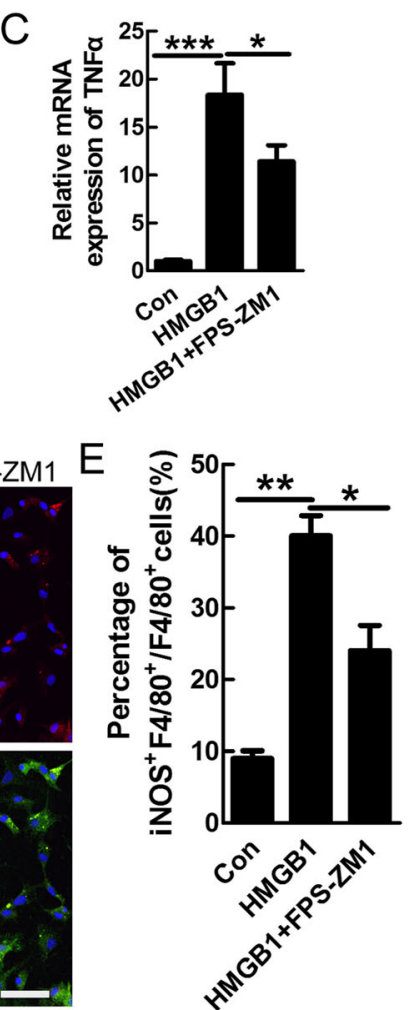

Relative protein expression

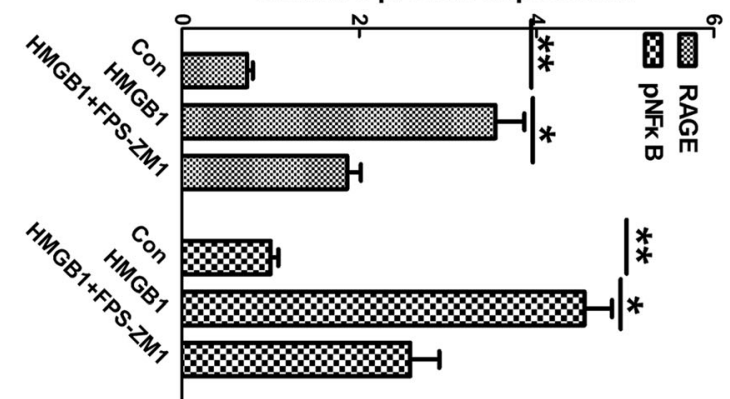

Fig. 5 Inhibiting RAGE-suppressed HMGB1- or SCl-induced pro-inflammatory polarization of macrophages/microglia. FPS-ZM1 (a RAGE-specific blocker) significantly reversed the increased mRNA of iNOS (a), CD86 (b), and TNFa (c) induced by HMGB1. $N=3,{ }^{*} P<0.05,{ }^{* *} P<0.01,{ }^{* * *} P<$ 0.001. d Representative images of double-staining of iNOS and F4/80 in microglia under normal condition, HMGB1 or HMGB1 plus FPS-ZM1 treatment. Scale bar $=30 \mu \mathrm{m}$. e Quantification of the percentage of iNOS-positive cells in F4/80-positive cells. Note that FPS-ZM1 significantly inhibited the increased number of iNOS-positive cells induced by HMGB1. $N=3,{ }^{*} P<0.05,{ }^{*} P<0.01$. $\mathbf{f}-\mathbf{g}$ Quantification of the expression levels of RAGE and $\mathrm{p}-\mathrm{NF}-\mathrm{KB}$ in microglia under different conditions. $\beta$-actin was used as a loading control. Data were expressed as mean $\pm \mathrm{SEM}$. $N=3$, ${ }^{*} P<0.05,{ }^{*} P<0.01$

above data indicated that inhibiting RAGE markedly reduced the extent of myelin loss after SCI.

\section{Inhibiting HMGB1-RAGE axis improved locomotor recovery after $\mathrm{SCl}$}

To test whether inhibiting HMGB1 or RAGE is beneficial to the locomotor recovery after SCI, BBB scoring and RHI assay were performed before injury and at 1,3 , 7, 10, 14, and 21 days after SCI. Compared with saline control, the glycyrrhizin or FPS-ZM1-treated rats showed significant higher BBB scores from 7 dpi $\left({ }^{*} P<\right.$ 0.05, Fig. 9a). Similarly, the glycyrrhizin or FPS-ZM1treated rats showed higher lift of the hind limbs from 7 dpi as evaluated by the RHI assay ( ${ }^{*} P<0.05$, Fig. $\left.9 b\right)$.

\section{Discussion}

Better and deeper understanding of the pathological mechanism after SCI is crucial for developing new treatment. The pro-inflammatory macrophages/microgliamediated pro-inflammatory response dominates the 

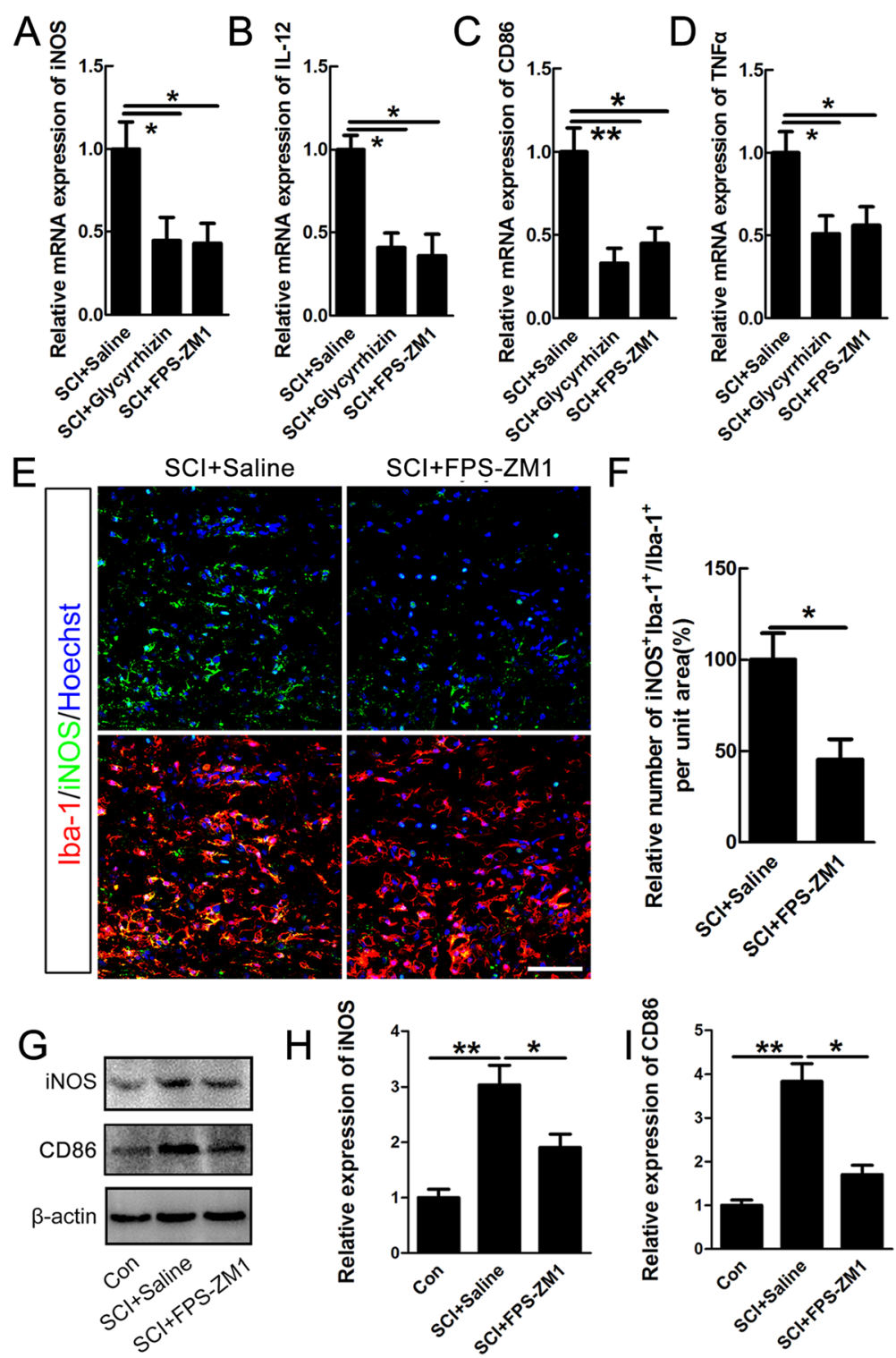

Fig. 6 Inhibiting HMGB1or RAGE reduced the numbers of pro-inflammatory macrophages/microglia after SCl. Quantification of pro-inflammatory mRNA of iNOS (a), IL-12 (b), CD86 (c), and TNFa (d) in saline-, glycyrrhizin-, or FPS-ZM1-treated rats at 14 dpi. Results are presented as mean \pm SEM. $N=3$ /group, ${ }^{*} P<0.05$. e Representative images of double-staining of iNOS and Iba-1 in saline- or FPS-ZM1-treated rats at $14 \mathrm{dpi}$. Scale bar $=80$ $\mu \mathrm{m}$. $\mathbf{f}$ Quantification of iNOS-positive microglia/macrophages in the bilateral areas $200 \mu \mathrm{m}$ rostral and caudal to the lesion site. Notice the decrease of iNOS-positive microglia/macrophages in FPS-ZM1-treated rats. Results are mean \pm SEM. $N=5 /$ group, ${ }^{*} P<0.05$. g-i Quantification of the expression levels of iNOS and CD86 in sham group, saline-, or FPS-ZM1-treated rats at 14 days after $\mathrm{SCl}$. $N=5$ /goup, ${ }^{*} P<0.05,{ }^{* *} P<0.01$

innate immune responses after SCI [6], but the mechanisms underlying this special process have been not fully elucidated. In the present study, we found that inhibiting the HMGB1-RAGE axis effectively prevented proinflammatory polarization of macrophages/microglia, decreased neuronal and myelin loss, and improved functional recovery after SCI.

Although it was found that HMGB1-RAGE does not aggravate neuronal inflammation [35], others demonstrated that inhibiting HMGB1 or RAGE could suppress the inflammation after injury [31, 34, 36-38]. The different results may be due to the different responses of neural cells to HMGB1 treatment and the different RAGE inhibitors used in different researches. However, the specific mechanism of HMGB1 or RAGE inhibition targeted on macrophages/microglia polarization had never been stated.

The data from genome-wide transcriptomics and proteomics indicated that the polarization of microglia and macrophage was not exactly the same [39]; we thus employed the terminology as pro-inflammatory (M1-like) 

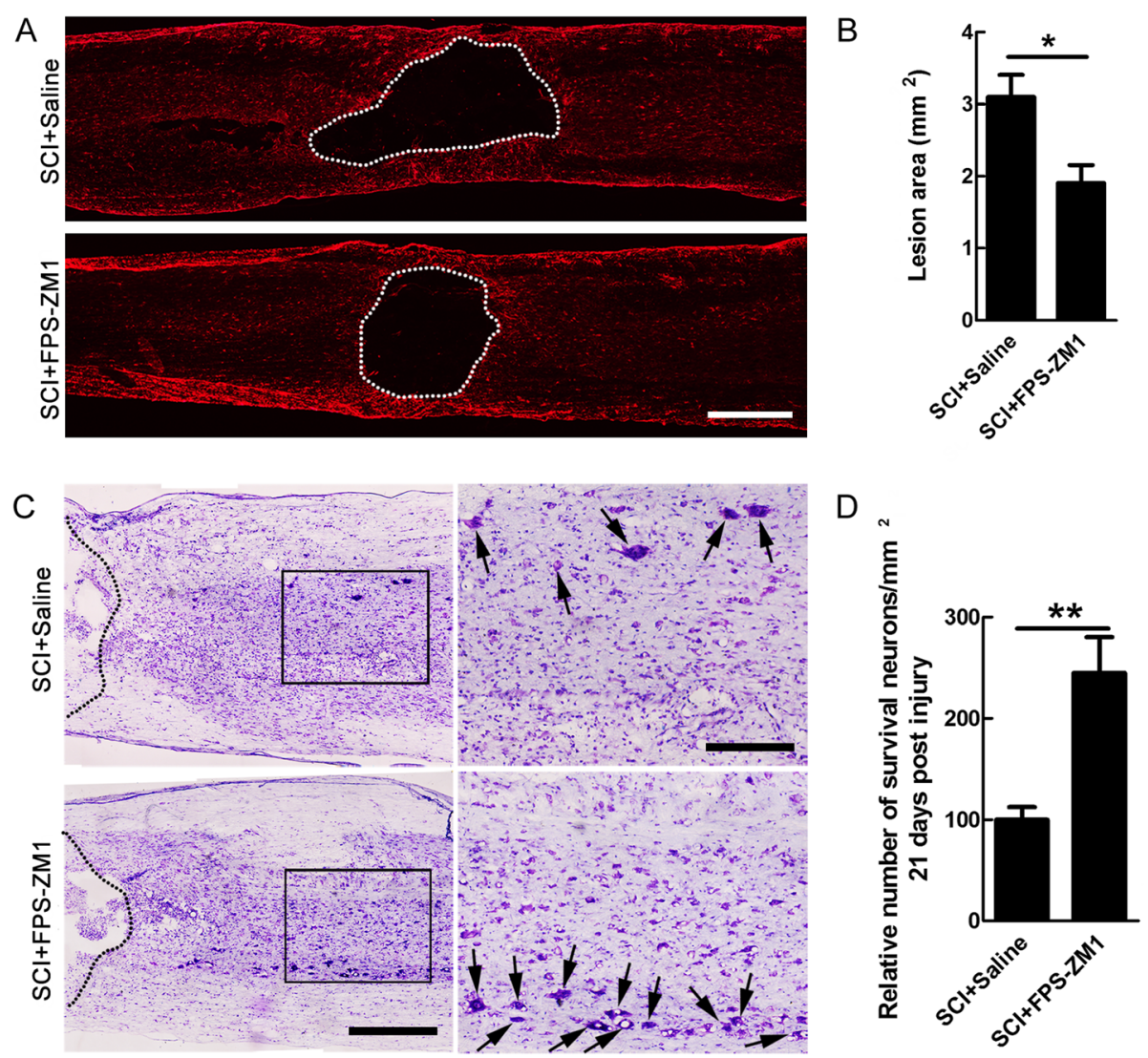

Fig. 7 The effects of FPS-ZM1 treatment on lesion area and neuronal survival after SCI. a Dashed lines indicate the lesion border defined by GFAP immunoreactivity in saline- or FPS-ZM1-treated rats at $21 \mathrm{dpi}$. Scale bar $=500 \mu \mathrm{m}$. b Quantification of lesion area. $N=6 /$ group, ${ }^{*} P<0.05$. c A representative Nissl-stained section of saline- or FPS-ZM1-treated rats at 21 dpi. Broken lines mark the lesion borders. Arrows indicate Nissl-stained neurons. Scale bar $=600 \mu \mathrm{m}$ in the left lower-magnification pictures and $100 \mu \mathrm{m}$ in the right higher-magnification pictures from the boxes of left. $\mathbf{d}$ Quantification of the numbers of Nissl-stained neurons at $21 \mathrm{dpi}$. Note that the numbers of Nissl-stained neurons was significantly increased by FPS-ZM1 treatment in rostral and caudal areas of $600 \mu \mathrm{m}$ adjacent to lesion. $N=6 /$ group, $* * P<0.01$

or anti-inflammatory (M2-like) phenotype as recommended [40]. Previous studies indicated the pro-inflammatory polarization of macrophages upon HMGB1 challenge [31], while HMGB1 was also reported to enhance the protumoral activities of anti-inflammatory macrophages [41]. However, the HMGB1-RAGE axis in polarization of macrophages/microglia after SCI has never been clearly defined. In this study, we first demonstrated that HMGB1 polarized pro-inflammatory microglia through RAGE-NF- $\mathrm{kB}$ pathway. Besides pro-inflammatory phenotype, we observed the effects of inhibiting HMGB1-RAGE on anti-inflammatory polarization of macrophages/microglia as shown in Additional file 2 . It will be an independent project to investigate its mechanism, because none of the molecular determinants of pro-anti-inflammatory polarization such as the PPAR, KLF, IRF, STAT, NF- $-\mathrm{B}$, and HIF families and miRNAs [42] could be excluded before exploration.

Besides the effects of HMGB1-RAGE inhibition on polarization of macrophages/microglia, we also observed its neuroprotective effects with focus on neuronal survival and myelin loss. Although it was reported that anti-RAGE antibody do damage to neuronal survival [43], we demonstrated that RAGE blockade could decrease neuronal loss by its inhibitor, FPS-ZM1. We proposed that the different effects might be attributed to the different inhibitors of RAGE. In addition, blocking RAGE was found to promote oligodendrocyte autophagy in spinal cord injury [44], which was similar with the results of the reduced myelin loss in our study. In this study, the direct function of RAGE inhibition on differentiation of oligodendrocyte precursor was precluded; we thus proposed that the reduced myelin loss by FPS-ZM1 in this study was probably due to the improved immune microenvironment of SCI. Remarkable functional recovery on BBB scale were observed from 8 to 9 to 18 in glycyrrhizin- or FPS-ZM1treated rats, and we speculated that it is probably due to the increased numbers of neurons and spared myelin, with other mechanisms cannot be excluded. 


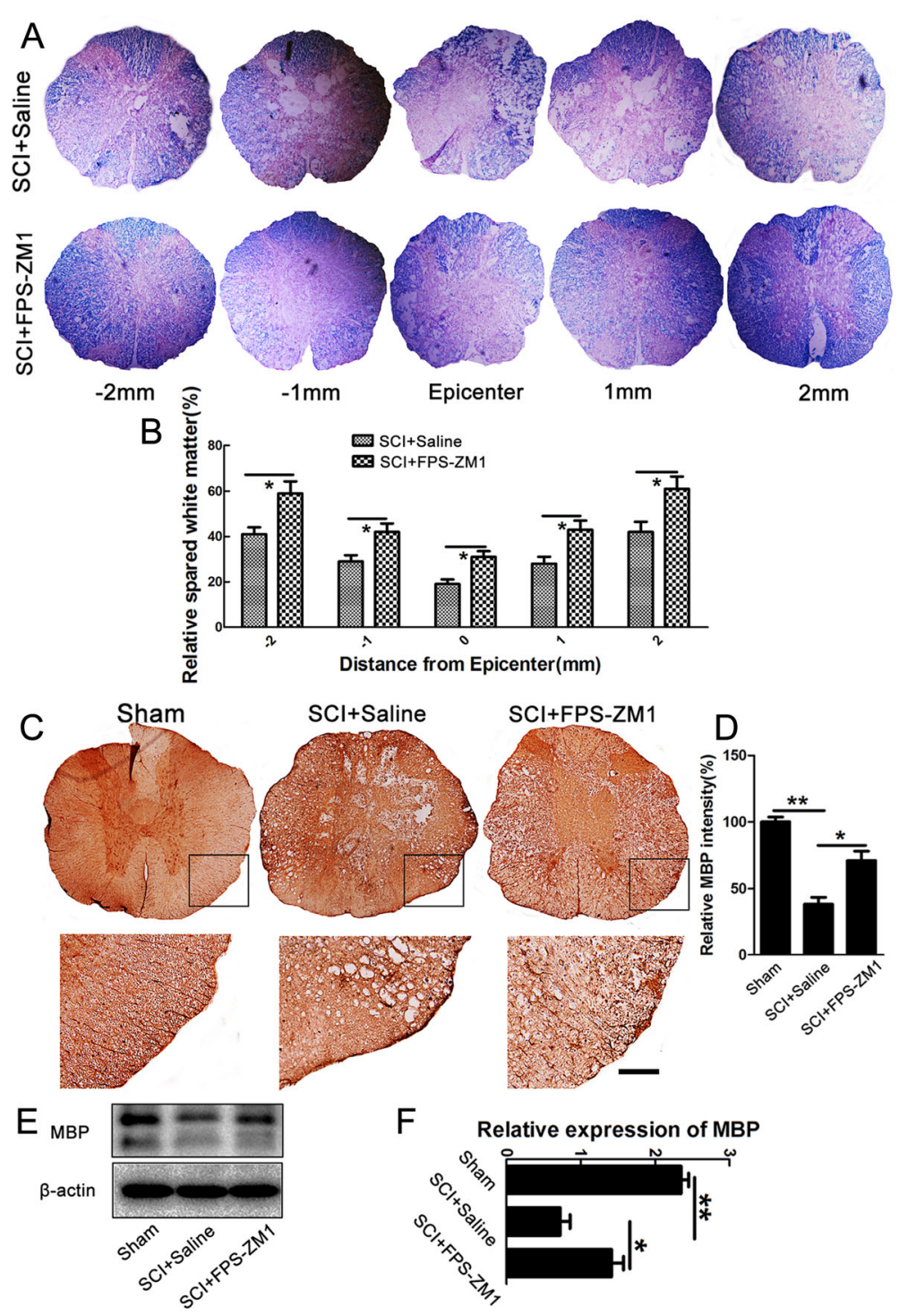

Fig. 8 Inhibiting RAGE by FPS-ZM1 reduced myelin loss after SCI. a Spinal cords at 21 days after injury were processed for Luxol fast blue in saline- or FPSZM1-treated rats. Transverse cryosections were selected $1000 \mu \mathrm{m}, 2000 \mu \mathrm{m}$ rostral and caudal to the lesion site, and the epicenter. b Quantification of spared myelin at $1000 \mu \mathrm{m}, 2000 \mu \mathrm{m}$ rostral and caudal to the lesion site, as well as at the epicenter. Data were expressed as mean \pm SEM of 6 rats, ${ }^{*} P<$ 0.05. c DAB staining of MBP in transverse sections at $21 \mathrm{dpi}$. Scale bar $=100 \mu \mathrm{m}$. $\mathbf{d}$ Quantification of MBP intensity. Note that FPS-ZM1 attenuated the reduction of MBP intensity in the white matter after injury. Data were expressed as mean \pm SEM of 6 rats, ${ }^{*} P<0.05$, ${ }^{*} P<0.01$. e-f Quantification of the protein expression level of MBP in sham group, saline-, or FPS-ZM1-treated rats at 21 days after SCI. N=6/group, ${ }^{*} P<0.05,{ }^{*} P<0.01$

HMGB1 has been demonstrated to be localized in the nucleus of astrocytes, microglia, neurons, and oligodendrocytes in naive spinal cord [45, 46], and its cellular localization was almost unchanged except for the cytoplasmic localization in Mac-1-positive macrophages in mice after SCI [31]. In this study, we observed that cytoplasmic HMGB1 was mainly localized in GFAP-positive astrocytes after SCI in rats, which was consistent with our previous result in SCI of mice [19]. In addition, cytoplasmic HMGB1 has been reported to be expressed by neurons after traumatic brain injury [47]. We speculated that the differences might be attributed to the species diversity and the different injury model. It has been definitively proved that HMGB1 functions as a DAMP to alert the innate immune system only when it is passively released by necrotic cells but not from cells undergoing apoptosis [13, 48]. As our previous study demonstrated that necroptotic astrocytes could release HMGB1 [19, 49], we speculated that the elevated serum HMGB1 was mostly originated from the HMGB1positive astrocytes, which might undergo necroptosis. We will test and verify this hypothesis by application of 

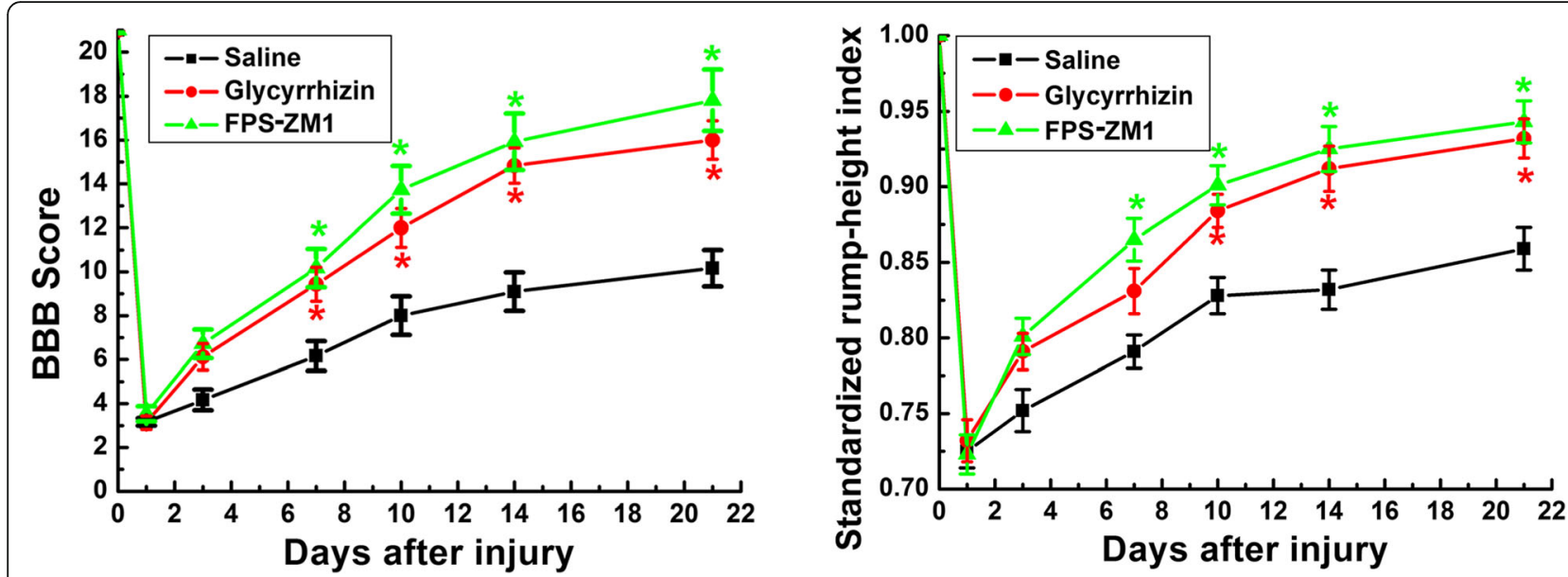

Fig. 9 Locomotion recovery improvement by glycyrrhizin or FPS-ZM1 treatment. a BBB scores at 1, 3, 7, 10, 14, and 21 days after SCI. The average BBB scores in glycyrrhizin- or FPS-ZM1-treated group were significantly higher than those in saline group at 7, 10, 14, and 21 days after injury. $\mathbf{b}$ $\mathrm{SRHI}$ values at $1,3,7,10,14$, and 21 days after $\mathrm{SCl}$. $\mathrm{N}=6 /$ group, ${ }^{*} P<0.05$

RIP3 and MLKL knock-out mice in future. In addition, it is important to prove antibody specificity first for immunohistochemistry as the guidelines showed [50]. Besides the validation by the manufacturer or the previous study [51], the specificity of antibodies of HMGB1, RAGE, and TLR4 was verified by western blot as shown in Additional file 3, and the full gels of other antibodies are shown in Additional file 4.

In recent years, several small molecules, derived either from natural sources or chemical synthesis explored as inhibitors of HMGB1, showed significant therapeutic importance [52]. Glycyrrhizin was one of the most widely used natural inhibitors, and studies have demonstrated that glycyrrhizin protected the liver, brain, and cardiovascular injury in experimental animals [53-55]. It was also reported that glycyrrhizin attenuated the transient spinal cord ischemic injury in rats via reducing inflammatory cytokines and inhibiting the release of HMGB1 [36]. In this study, the inhibition of release of HMGB1 was not found by glycyrrhizin treatment after SCI (data not shown), and we proposed that glycyrrhizin could inhibit the receptor activation of HMGB1 as shown by the decreased percentage of RAGE-positive cells in F4/80-positive cells after SCI. Besides the small molecules, anti-HMGB1 monoclonal antibody (mAb) had been demonstrated to neutralize the released HMGB1, prevent the inflammation cascade, and afford a therapeutic effect in ischemic brain injury, traumatic brain injury, Parkinson's disease, and SCI [14, 56-59]. One research showed that acute intraperitoneal use of HMGB1 neutralizing antibody failed to provide protection after SCI [31], which suggested that small molecule inhibitors of HMGB1 such as glycyrrhizin may be preferable to blocking antibodies since these molecules can bypass the blood-spinal cord barrier more effectively. On the other hand, beneficial effects of anti-HMGB1 mAb for SCI depended on a proper time window for drug administration as demonstrated previously [59]. In addition, it was reported that prior treatment with antiHMGB-1 antibody enhanced human neural stem cell transplantation-mediated functional recovery after SCI, and this effect was mediated by increased synaptic connectivity between increased preserved host neurons and transplant-derived neurons [60], which was similar as increased neuronal survival by FPS-ZM1 in this study.

Although at least 14 different receptor systems have been reported to be engaged by extracellular HMGB1, only TLR4 and RAGE have so far been extensively studied in carefully defined molecular systems and confirmed in a substantial number of studies to act as specific HMGB1 receptors [32]. We thus examined the changes of these two receptors, and our data of qPCR and immunostaining suggested that TLR4 was unlikely to be involved in HMGB1-induced pro-inflammatory polarization of microglia. By application of FPS-ZM1, we demonstrated that RAGE contributed to the pro-inflammatory polarization of microglia upon HMGB1 treatment. Nevertheless, RAGE was reported to be expressed by neurons, glia, and endothelial cells [61-64]. We therefore cannot exclude the possibility that the activation of RAGE in neurons or endothelial cells attributed to the neuroprotective effect of HMGB1 inhibition.

\section{Conclusions}

This study demonstrated that inhibiting HMGB1-RAGE axis prevented pro-inflammatory macrophages/microglia polarization and afforded neuroprotection after SCI in rats. Thus, therapies targeting HMGB1-RAGE axis might be a prospective clinical treatment for SCI. 


\section{Supplementary information}

Supplementary information accompanies this paper at https://doi.org/10. 1186/s12974-020-01973-4.

Additional file 1: Figure S1. Double-staining of Iba-1/TLR4 at $5 \mathrm{dpi}$. Note that about 17\% TLR4-positive cells were Iba-1 positive. Scale bar = $50 \mu \mathrm{m}$.

Additional file 2: Figure S2. Inhibiting HMGB1 or RAGE increased the numbers of anti-inflammatory macrophages/microglia after SCl. Quantification of anti-inflammatory-associated mRNA transcripts of Arginase1 (A) Ym1 (B), IL-4 (C), IL-10 (D) in saline-, FPS-ZM1- or Glycyrrhizin-treated rats at $14 \mathrm{dpi}$. Results are presented as mean \pm SEM of $n=3,{ }^{*} P<0.05$. (E) Double-staining of Arginase 1 and Iba-1 in saline- or FPS-ZM1-treated rats at $14 \mathrm{dpi}$. Scale bar $=80 \mu \mathrm{m}$. (F) Quantification of the numbers of Arginase1-positive microglia/macrophages in lesion epicenter. Results are presented as mean \pm SEM. $N=5 /$ group, ${ }^{*} P<0.05$.

Additional file 3: Figure S3. Western blot of HMGB1, RAGE, and TLR4 The specificity of antibodies of HMGB1, RAGE and TLR4 was verified by western blot. Note that the antibodies (HMGB1, RAGE, and TLR4) recognize only one antigen respectively, and each band is of the appropriate molecular weight.

Additional file 4: Figure S4. Full gels of RAGE, pNF-kB, iNOS, CD86 and $\beta$-actin.

\section{Abbreviations}

SCI: Spinal cord injury; qRT-PCR: Quantitative reverse transcription polymerase chain reaction; DAMPs: Damage-associated molecular patterns; HMGB1: High mobility group box 1; RAGE: Receptor for advanced glycation end products; NF-kB: Nuclear factor-kappa B; LFB: Luxol fast blue; BBB: BassoBeattie-Bresnahan; RHI: Rump-height index

\section{Acknowledgments}

We thank the support from the Department of Neurology, The Second Affiliated Hospital, Xi'an Jiaotong University, and the Institute of Neurosciences, Fourth Military Medical University.

\section{Authors' contributions}

Hong Fan, Hai-Bin Tang, and Zhe Chen wrote the paper and performed the most of experiments. Hu-Qing Wang, Lei Zhang, Yu Jiang, Tao Li, Cai-Feng Yang, Xiao-Ya Wang, and Xia Li contributed morphological study and analyzed the data. Sheng-Xi Wu and Gui-Lian Zhang designed the research and revised the manuscript.

\section{Funding}

This work was supported by grants from the National Natural Science Foundation of China (NSFC, Grant code: 81701204) and project funded by China Postdoctoral Science Foundation (Grant code: 2018M643703, 2020T130526) to Hong Fan, NSFC (Grant code: 81971116) and Shaanxi Science Research Project (Grant code: 2019ZDLSF01-04) to Gui-Lian Zhang, and Research Project of Xi'an Health Commission (Grant code: J201902019) to Hai-Bin Tang. This work was supported by the Fundamental Research Funds for the Central Universities of China (xzy012020063).

\section{Availability of data and materials}

The datasets generated and/or analyzed in the current study are available from the corresponding author on reasonable request.

\section{Ethics approval and consent to participate}

All protocols were approved by the Animal Care and Use Committee of Xi'an Jiaotong University and conformed to the Guide for the Care and Use of Laboratory Animals by the National Institutes of Health, USA.

\section{Consent for publication}

Not applicable

\section{Competing interests}

The authors declare no conflicts of interest.

\section{Author details}

'Department of Neurology, The Second Affiliated Hospital of Xi'an Jiaotong University, Xi'an 710004, Shaanxi, China. ${ }^{2}$ Institute of Neurosciences, Fourth Military Medical University, Xi'an 710032, Shaanxi, China. ${ }^{3}$ Department of Laboratory Medicine, Xi'an Central Hospital, Xi'an Jiaotong University, 161 Xi Wu Road, Xi'an 710003, Shaanxi, China. ${ }^{4}$ Xi'an Jiaotong University Health Science Center, Xi'an Jiaotong University, Xi'an 710061, Shaanxi, China. ${ }^{5}$ Department of Nephrology, The Second Affiliated Hospital of Xi'an Jiaotong University, Xi'an 710004, Shaanxi, China.

Received: 21 May 2020 Accepted: 30 September 2020

Published online: 09 October 2020

\section{References}

1. Ahuja CS, Wilson JR, Nori S, Kotter MRN, Druschel C, Curt A, Fehlings MG. Traumatic spinal cord injury. Nat Rev Dis Primers. 2017;3:17018.

2. Chen J, Shifman MI. Inhibition of neogenin promotes neuronal survival and improved behavior recovery after spinal cord injury. Neuroscience. 2019;408: 430-47.

3. Li Q, Houdayer T, Liu S, Belegu V. Induced neural activity promotes an oligodendroglia regenerative response in the injured spinal cord and improves motor function after spinal cord injury. J Neurotrauma. 2017;34: 3351-61.

4. Fan $H$, Tang HB, Shan LQ, Liu SC, Huang DG, Chen X, Chen Z, Yang M, Yin $\mathrm{XH}$, Yang H, Hao DJ. Quercetin prevents necroptosis of oligodendrocytes by inhibiting macrophages/microglia polarization to M1 phenotype after spinal cord injury in rats. J Neuroinflammation. 2019;16:206.

5. Chen H, Ji H, Zhang M, Liu Z, Lao L, Deng C, Chen J, Zhong G. An agonist of the protective factor SIRT1 improves functional recovery and promotes neuronal survival by attenuating inflammation after spinal cord injury. J Neurosci. 2017;37:2916-30.

6. Gensel JC, Zhang B. Macrophage activation and its role in repair and pathology after spinal cord injury. Brain Res. 1619;2015:1-11.

7. Zhou X, He X, Ren Y. Function of microglia and macrophages in secondary damage after spinal cord injury. Neural Regen Res. 2014;9:1787-95.

8. Gulke E, Gelderblom M, Magnus T. Danger signals in stroke and their role on microglia activation after ischemia. Ther Adv Neurol Disord. 2018;11: 1756286418774254

9. Nagata S. Apoptosis and clearance of apoptotic cells. Annu Rev Immunol. 2018;36:489-517.

10. Zheng Y, Gardner SE, Clarke MC. Cell death, damage-associated molecular patterns, and sterile inflammation in cardiovascular disease. Arterioscler Thromb Vasc Biol. 2011;31:2781-6.

11. Bajwa $\mathrm{E}$, Pointer $\mathrm{CB}$, Klegeris $\mathrm{A}$. The role of mitochondrial damageassociated molecular patterns in chronic neuroinflammation. Mediat Inflamm. 2019:2019:4050796.

12. Paudel YN, Shaikh MF, Chakraborti A, Kumari Y, Aledo-Serrano A, Aleksovska K, Alvim MKM, Othman I. HMGB1: A common biomarker and potential target for $\mathrm{TBI}$, neuroinflammation, epilepsy, and cognitive dysfunction. Front Neurosci. 2018;12:628.

13. Scaffidi P, Misteli T, Bianchi ME. Release of chromatin protein HMGB1 by necrotic cells triggers inflammation. Nature. 2002;418:191-5.

14. Okuma Y, Liu K, Wake H, Zhang J, Maruo T, Date I, Yoshino T, Ohtsuka A, Otani N, Tomura S, et al. Anti-high mobility group box-1 antibody therapy for traumatic brain injury. Ann Neurol. 2012;72:373-84.

15. Kim SW, Lee H, Lee HK, Kim ID, Lee JK. Neutrophil extracellular trap induced by HMGB1 exacerbates damages in the ischemic brain. Acta Neuropathol Commun. 2019;7:94.

16. Walker LE, Frigerio F, Ravizza T, Ricci E, Tse K, Jenkins RE, Sills GJ, Jorgensen A Porcu L, Thippeswamy $T$, et al. Molecular isoforms of high-mobility group box 1 are mechanistic biomarkers for epilepsy. J Clin Invest. 2017:127:2118-32.

17. Malhotra S, Fissolo N, Tintore M, Wing AC, Castillo J, Vidal-Jordana A, Montalban X, Comabella M. Role of high mobility group box protein 1 (HMGB1) in peripheral blood from patients with multiple sclerosis. J Neuroinflammation. 2015;12:48.

18. Papatheodorou A, Stein A, Bank M, Sison CP, Gibbs K, Davies P, Bloom O. Highmobility group box 1 (HMGB1) is elevated systemically in persons with acute or chronic traumatic spinal cord injury. J Neurotrauma. 2017;34:746-54.

19. Fan H, Zhang K, Shan L, Kuang F, Chen K, Zhu K, Ma H, Ju G, Wang YZ. Reactive astrocytes undergo M1 microglia/macrohpages-induced necroptosis in spinal cord injury. Mol Neurodegener. 2016;11:14. 
20. Hong Y, Shen C, Yin Q, Sun M, Ma Y, Liu X. Effects of RAGE-Specific Inhibitor FPS-ZM1 on amyloid-beta metabolism and AGEs-induced inflammation and oxidative stress in rat hippocampus. Neurochem Res. 2016:41:1192-9.

21. Deane R, Singh I, Sagare AP, Bell RD, Ross NT, LaRue B, Love R, Perry S, Paquette N, Deane RJ, et al. A multimodal RAGE-specific inhibitor reduces amyloid beta-mediated brain disorder in a mouse model of Alzheimer disease. J Clin Invest. 2012;122:1377-92.

22. Li Y, Sun F, Jing Z, Wang X, Hua X, Wan L. Glycyrrhizic acid exerts antiinflammatory effect to improve cerebral vasospasm secondary to subarachnoid hemorrhage in a rat model. Neurol Res. 2017;39:727-32.

23. Plemel JR, Duncan G, Chen KW, Shannon C, Park S, Sparling JS, Tetzlaff W. A graded forceps crush spinal cord injury model in mice. J Neurotrauma. 2008;25:350-70.

24. Fan $\mathrm{H}$, Chen $\mathrm{K}$, Duan $\mathrm{L}$, Wang $\mathrm{YZ}$, Ju G. Beneficial effects of early hemostasis on spinal cord injury in the rat. Spinal Cord. 2016;54:924-32.

25. Basso DM, Beattie MS, Bresnahan JC. A sensitive and reliable locomotor rating scale for open field testing in rats. J Neurotrauma. 1995;12:1-21.

26. Sedy J, Urdzikova L, Jendelova P, Sykova E. Methods for behavioral testing of spinal cord injured rats. Neurosci Biobehav Rev. 2008;32:550-80.

27. Fan $H$, Liu $X$, Tang HB, Xiao $P$, Wang $Y Z$, Ju G. Protective effects of batroxobin on spinal cord injury in rats. Neurosci Bull. 2013;29:501-8.

28. Coggeshall RE, Lekan HA. Methods for determining numbers of cells and synapses: a case for more uniform standards of review. J Comp Neurol. 1996:364:6-15

29. Coggeshall RE, La Forte R, Klein CM. Calibration of methods for determining numbers of dorsal root ganglion cells. J Neurosci Methods. 1990;35:187-94.

30. Frank MG, Weber MD, Watkins LR, Maier SF. Stress sounds the alarmin: the role of the danger-associated molecular pattern HMGB1 in stress-induced neuroinflammatory priming. Brain Behav Immun. 2015;48:1-7.

31. Kigerl KA, Lai W, Wallace LM, Yang H, Popovich PG. High mobility group box-1 (HMGB1) is increased in injured mouse spinal cord and can elicit neurotoxic inflammation. Brain Behav Immun. 2018;72:22-33.

32. Andersson $U$, Yang $H$, Harris $H$. High-mobility group box 1 protein (HMGB1) operates as an alarmin outside as well as inside cells. Semin Immunol. 2018.

33. Pei JP, Fan LH, Nan K, Li J, Dang XQ, Wang KZ. HSYA alleviates secondary neuronal death through attenuating oxidative stress, inflammatory response, and neural apoptosis in SD rat spinal cord compression injury. J Neuroinflammation. 2017;14:97.

34. Chen KB, Uchida K, Nakajima H, Yayama T, Hirai T, Rodriguez Guerrero A, Kobayashi S, Ma WY, Liu SY, Zhu P, Baba H. High-mobility group box-1 and its receptors contribute to proinflammatory response in the acute phase of spinal cord injury in rats. Spine (Phila Pa 1976). 2011;36:2122-9.

35. Wang H, Mei X, Cao Y, Liu C, Zhao Z, Guo Z, Bi Y, Shen Z, Yuan Y, Guo Y, et al. HMGB1/advanced glycation end products (RAGE) does not aggravate inflammation but promote endogenous neural stem cells differentiation in spinal cord injury. Sci Rep. 2017;7:10332

36. Gong G, Yuan LB, Hu L, Wu W, Yin L, Hou JL, Liu YH, Zhou LS. Glycyrrhizin attenuates rat ischemic spinal cord injury by suppressing inflammatory cytokines and HMGB1. Acta Pharmacol Sin. 2012;33:11-8.

37. Yang H, Liu H, Zeng Q, Imperato GH, Addorisio ME, Li J, He M, Cheng KF, Al-Abed Y, Harris HE, et al. Inhibition of HMGB1/RAGE-mediated endocytosis by HMGB1 antagonist box A, anti-HMGB1 antibodies, and cholinergic agonists suppresses inflammation. Mol Med. 2019;25:13.

38. Zhang S, Hu L, Jiang J, Li H, Wu Q, Ooi K, Wang J, Feng Y, Zhu D, Xia C. HMGB1/RAGE axis mediates stress-induced RVLM neuroinflammation in mice via impairing mitophagy flux in microglia. J Neuroinflammation. 2020;17:15.

39. Ransohoff RM. A polarizing question: do M1 and M2 microglia exist? Nat Neurosci. 2016;19:987-91.

40. Lan X, Han X, Li Q, Yang QW, Wang J. Modulators of microglial activation and polarization after intracerebral haemorrhage. Nat Rev Neurol. 2017;13: 420-33.

41. Rojas A, Delgado-Lopez F, Perez-Castro R, Gonzalez I, Romero J, Rojas I, Araya P, Anazco C, Morales E, Llanos J. HMGB1 enhances the protumoral activities of M2 macrophages by a RAGE-dependent mechanism. Tumour Biol. 2016:37:3321-9.

42. Wang $\mathrm{N}$, Liang $\mathrm{H}$, Zen $\mathrm{K}$. Molecular mechanisms that influence the macrophage m1-m2 polarization balance. Front Immunol. 2014;5:614.

43. Wang H, Zhao Z, Liu C, Guo Z, Yuan Y, Zhao H, Zhou Z, Mei X. Receptor for advanced glycation end-products (RAGE) blockade do damage to neuronal survival via disrupting Wnt/beta-catenin signaling in spinal cord injury. Neurochem Res. 2018;43:1405-12.

44. Mei X, Wang H, Zhang H, Liu C, Guo Z, Wang Y, Yuan Y, Zhao Z, Li D, Tang P. Blockade of receptor for advanced glycation end products promotes oligodendrocyte autophagy in spinal cord injury. Neurosci Lett. 2019;698:198-203.

45. Dong Y, Gu Y, Huan Y, Wang Y, Liu Y, Liu M, Ding F, Gu X, Wang Y. HMGB1 protein does not mediate the inflammatory response in spontaneous spinal cord regeneration: a hint for CNS regeneration. J Biol Chem. 2013;288: 18204-18.

46. Kikuchi K, Uchikado H, Miura N, Morimoto Y, Ito T, Tancharoen S, Miyata K, Sakamoto R, Kikuchi C, lida N, et al. HMGB1 as a therapeutic target in spinal cord injury: a hypothesis for novel therapy development. Exp Ther Med. 2011;2:767-70.

47. Laird MD, Shields JS, Sukumari-Ramesh S, Kimbler DE, Fessler RD, Shakir B, Youssef P, Yanasak N, Vender JR, Dhandapani KM. High mobility group box protein-1 promotes cerebral edema after traumatic brain injury via activation of toll-like receptor 4. Glia. 2014;62:26-38.

48. Kono H, Rock KL. How dying cells alert the immune system to danger. Nat Rev Immunol. 2008;8:279-89.

49. Fan H, Tang HB, Kang J, Shan L, Song H, Zhu K, Wang J, Ju G, Wang YZ. Involvement of endoplasmic reticulum stress in the necroptosis of microglia/ macrophages after spinal cord injury. Neuroscience. 2015;311:362-73.

50. Saper CB, Sawchenko PE. Magic peptides, magic antibodies: guidelines for appropriate controls for immunohistochemistry. J Comp Neurol. 2003;465:161-3.

51. Qian QQ, Zhang X, Wang YW, Xu JW, Dong HQ, Li NN, Qian YN, Gui B. Proinflammatory role of high-mobility group box-1 on brain mast cells via the RAGE/NF-kappaB pathway. J Neurochem. 2019;151:595-607.

52. Musumeci D, Roviello GN, Montesarchio D. An overview on HMGB1 inhibitors as potential therapeutic agents in HMGB1-related pathologies. Pharmacol Ther. 2014;141:347-57.

53. Ohnishi M, Katsuki H, Fukutomi C, Takahashi M, Motomura M, Fukunaga M, Matsuoka Y, Isohama Y, Izumi Y, Kume T, et al. HMGB1 inhibitor glycyrrhizin attenuates intracerebral hemorrhage-induced injury in rats. Neuropharmacology. 2011;61:975-80.

54. Ogiku M, Kono H, Hara M, Tsuchiya M, Fujii H. Glycyrrhizin prevents liver injury by inhibition of high-mobility group box 1 production by Kupffer cells after ischemia-reperfusion in rats. J Pharmacol Exp Ther. 2011;339:93-8.

55. Zhai $C L$, Zhang MQ, Zhang Y, Xu HX, Wang JM, An GP, Wang YY, Li L. Glycyrrhizin protects rat heart against ischemia-reperfusion injury through blockade of HMGB1-dependent phospho-JNK/Bax pathway. Acta Pharmacol Sin. 2012;33:1477-87.

56. Sasaki T, Liu K, Agari T, Yasuhara T, Morimoto J, Okazaki M, Takeuchi H, Toyoshima A, Sasada S, Shinko A, et al. Anti-high mobility group box 1 antibody exerts neuroprotection in a rat model of Parkinson's disease. Exp Neurol. 2016;275(Pt 1):220-31.

57. Haruma J, Teshigawara K, Hishikawa T, Wang D, Liu K, Wake H, Mori S, Takahashi HK, Sugiu K, Date I, Nishibori M. Anti-high mobility group box-1 (HMGB1) antibody attenuates delayed cerebral vasospasm and brain injury after subarachnoid hemorrhage in rats. Sci Rep. 2016;6:37755.

58. Zhang J, Takahashi HK, Liu K, Wake H, Liu R, Maruo T, Date I, Yoshino T, Ohtsuka A, Mori S, Nishibori M. Anti-high mobility group box-1 monoclonal antibody protects the blood-brain barrier from ischemia-induced disruption in rats. Stroke. 2011;42:1420-8.

59. Nakajo M, Uezono N, Nakashima H, Wake H, Komiya S, Nishibori M, Nakashima K. Therapeutic time window of anti-high mobility group box-1 antibody administration in mouse model of spinal cord injury. Neurosci Res. 2019;141:63-70.

60. Uezono N, Zhu Y, Fujimoto Y, Yasui T, Matsuda T, Nakajo M, Abematsu M, Setoguchi T, Mori S, Takahashi HK, et al. Prior treatment with anti-high mobility group box-1 antibody boosts human neural stem cell transplantation-mediated functional recovery after spinal cord injury. Stem Cells. 2018;36:737-50.

61. Qiu J, Nishimura M, Wang Y, Sims JR, Qiu S, Savitz SI, Salomone S, Moskowitz MA. Early release of HMGB-1 from neurons after the onset of brain ischemia. J Cereb Blood Flow Metab. 2008;28:927-38.

62. Qin J, Goswami R, Dawson S, Dawson G. Expression of the receptor for advanced glycation end products in oligodendrocytes in response to oxidative stress. J Neurosci Res. 2008;86:2414-22.

63. Li W, Xu H, Hu Y, He P, Ni Z, Xu H, Zhang Z, Dai H. Edaravone protected human brain microvascular endothelial cells from methylglyoxal-induced injury by inhibiting AGEs/RAGE/oxidative stress. PLoS One. 2013;8:e76025. 
64. Kim J, Waldvogel HJ, Faull RL, Curtis MA, Nicholson LF. The RAGE receptor and its ligands are highly expressed in astrocytes in a grade-dependant manner in the striatum and subependymal layer in Huntington's disease. J Neurochem. 2015;134:927-42.

\section{Publisher's Note}

Springer Nature remains neutral with regard to jurisdictional claims in published maps and institutional affiliations.

- fast, convenient online submission

- thorough peer review by experienced researchers in your field

- rapid publication on acceptance

- support for research data, including large and complex data types

- gold Open Access which fosters wider collaboration and increased citations

- maximum visibility for your research: over $100 \mathrm{M}$ website views per year

At BMC, research is always in progress. 